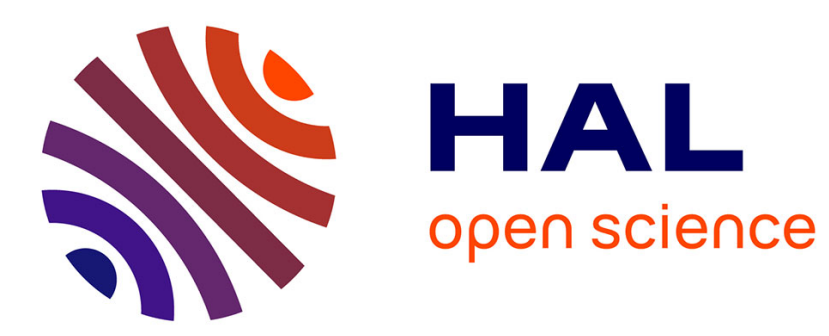

\title{
QUADRUPOLE INTERACTIONS IN RARE-EARTH INTERMETALLIC COMPOUNDS
}

\author{
P Morin, D. Schmitt
}

\section{To cite this version:}

P Morin, D. Schmitt. QUADRUPOLE INTERACTIONS IN RARE-EARTH INTERMETALLIC COMPOUNDS. Journal de Physique Colloques, 1988, 49 (C8), pp.C8-321-C8-325. 10.1051/jphyscol:19888144 . jpa-00228290

\section{HAL Id: jpa-00228290 https://hal.science/jpa-00228290}

Submitted on 1 Jan 1988

HAL is a multi-disciplinary open access archive for the deposit and dissemination of scientific research documents, whether they are published or not. The documents may come from teaching and research institutions in France or abroad, or from public or private research centers.
L'archive ouverte pluridisciplinaire HAL, est destinée au dépôt et à la diffusion de documents scientifiques de niveau recherche, publiés ou non, émanant des établissements d'enseignement et de recherche français ou étrangers, des laboratoires publics ou privés. 


\title{
QUADRUPOLE INTERACTIONS IN RARE-EARTH INTERMETALLIC COMPOUNDS
}

\author{
P. Morin and D. Schmitt \\ Laboratoire Louis Néel, C.N.R.S., 166X, 38042 Grenoble Cedex, France
}

\begin{abstract}
A survey of the role played by the one-ion magnetoelastic and two-ion quadrupolar interactions on the magnetic and elastic properties of the rare-earth intermetallic compounds is given. These couplings are present in numerous systems and manifest themselves through a great variety of effects.
\end{abstract}

The study of the multipolar interactions concerning the $4 \mathrm{f}$ shell has known a great development about rare earth intermetallic compounds [1]. It involves the complex cohabitation of i) the 4 f electron-lattice coupling which gives rise to the usual crystal field acting on the rare-earth, as well as to the one-ion magnetoelastic coupling; ii) the bilinear and quadrupolar pair interactions between different sites leading to possible long-range orderings. The aim of this review is to present a survey of the experimental manifestations of the one-ion magnetoelastic and the two-ion quadrupolar interactions; both couplings involve the orbital character of the $4 f$ shell through its quadrupolar components.

\section{The Hamiltonian}

The existence of non quenched orbital moments in the rare earth ions leads to anisotropic magnetic properties. A classical description of these properties is no longer valid. In a quantum formalism, the $4 \mathrm{f}$ shell is represented by wavefunctions expanded in the $\left|J, M_{J}\right\rangle$ basis. The appropriate Hamiltonian, $\mathcal{H}$ which determines these wavefunctions, includes several terms associated with the various interactions in presence. The first term to be considered is the Crystalline Electric Field (CEF) Hamiltonian which may be expressed as a function of linear combinations $O_{\mu}$ of the Stevens operators [2]:

$$
\mathcal{H}_{\mathrm{CEF}}=\sum_{\mu} A_{\mu} O_{\mu}
$$

where the $A_{\mu}$ 's are the CEF parameters. The number and the detailed form of the $O_{\mu}{ }^{\prime} \mathrm{s}$ depend on the point symmetry of the rare earth site under consideration. This CEF term may be modulated by the strain through the one-ion Hamiltonian $\mathcal{H}_{\mathrm{ME}}$ :

$$
\mathcal{H}_{\mathrm{ME}}=\sum_{\mu} B^{\mu} \varepsilon_{\mu} Q_{\mu} .
$$

In this expression, the $Q_{\mu}{ }^{\prime}$ s represent proper combinations of the second-order Stevens operators, which are coupled with the corresponding symmetrized strain $\varepsilon_{\mu}$ through the magnetoelastic coefficients $B^{\mu}$. Here we neglect any anharmonic or two-ion magnetoelastic coupling which may however play a role in some particular cases.

The next term in $\mathcal{H}$ involves the total angular momentum $\mathbf{J}$ through the Zeeman coupling $-g_{J} \mu_{\mathrm{B}} \mathbf{H}$. J as well as the isotropic bilinear Heisenberg-type Hamiltonian:

$$
\mathcal{H}_{\mathrm{B}}=-\left(g_{J} \mu_{\mathrm{B}}\right)^{2} n\langle\mathbf{J}\rangle . \mathbf{J}=-g_{J} \mu_{\mathrm{B}} n \mathbf{M} . \mathbf{J} .
$$

Within the mean field approximation (MFA), $n$ is the bilinear exchange parameter. In the same way, the $4 f$ quadrupoles may be coupled between each other through direct or indirect mechanisms (see paragraph 4), leading to the general expression in the MFA:

$$
\mathcal{H}_{Q}=-\sum_{\mu} K^{\mu}\left\langle Q_{\mu}\right\rangle Q_{\mu}
$$

where the $K^{\mu \prime}$ s are the two-ion quadrupolar parameters.

\section{Treatment of the Hamiltonian}

There is two ways to use the above Hamiltonian to describe the magnetic properties. First a diagonalization can be performed in a self-consistent manner. This allows us to determine the magnetization and magnetostriction processes, in particular in the ordered phase or in large external stresses (magnetic field or external pressure). The second possibility is to apply perturbation theory in order to describe both magnetic and magnetoelastic properties in the nonordered phase and in small external stresses. An analytical expansion of the free energy $F^{\mu}$ is then derived as a function of the magnetic field $H$ and of the strain $\varepsilon_{\mu}$, for a given symmetry:

$$
\begin{aligned}
F^{\mu}=F_{0}^{\mu} & -\frac{1}{2} \chi_{0}(H+n M)^{2}-\frac{1}{2} \chi_{\mu}\left(B^{\mu} \varepsilon_{\mu}+K^{\mu} Q_{\mu}\right)^{2} \\
& -\chi_{\mu}^{(2)}\left(B^{\mu} \varepsilon_{\mu}+K^{\mu} Q_{\mu}\right)(H+n M)^{2} \\
& -\frac{1}{4} \chi_{\mu}^{(3)}(H+n M)^{2} \\
& +\frac{1}{2} c_{0}^{\mu} \varepsilon_{\mu}^{2}+\frac{1}{2} n M^{2}+\frac{1}{2} K^{\mu} Q_{\mu}^{2}
\end{aligned}
$$


This expression involves several CEF single-ion susceptibilities $\chi_{\mu}^{(n)}$, which connect specifically the various thermodynamical variables $M$ and $Q_{\mu}$ to $H$ and $\varepsilon_{\mu}$; this will lead to specific experiments (see below).

The low temperature behaviour of these susceptibilities appears to be dominated by CEF effects. Especially they provide us with informations about the magnetic and quadrupolar character of the lowest CEF levels, giving rise to Curie-type, van Vleck-type or more complex behaviours as it will be shown below.

\section{Experimental manifestations}

The magnetoelastic and quadrupolar couplings manifest themselves through a great variety of effects. The most spectacular evidence is the quadrupolar ordering at which the quadrupoles become coherently aligned without any simultaneous magnetic ordering. This has been observed in a few cubic rare earth compounds, where, because of the symmetry, no quadrupolar component exists spontaneously. Due to the magnetoelastic coupling, a symmetry lowering may then occur and reflect the symmetry of the quadrupole arrangement.

Large orbital couplings and weak bilinear interactions are necessary to reveal such situations, which thus appear at each end of the lanthanide sequence: $\mathrm{TmZn}$ [3], TmCd [4], $\mathrm{CeAg}[5]$ and $\mathrm{PrCu}_{2}$ [6] order ferroquadrupolarly, while $\mathrm{PrPb}_{3}[7], \mathrm{CeB}_{6}[8]$ and $\mathrm{TmGa}_{3}$ [9] exhibit antiferroquadrupolar ordering. In these latter two compounds, the non-collinear arrangement of the $4 \mathrm{f}$ quadrupoles is evidenced not directly, but either through the ferrimagnetic spin structure induced by an external magnetic field [10], or through the multiaxial spin arrangement which takes place at a lower temperature [11].

In the presence of competing bilinear and quadrupolar interactions, different magnetic and/or quadrupolar phases may occur according to the relative strength of the associated coupling parameters $n$ and $G^{\mu}$. In cubic symmetry for example, a complex phase diagram can be obtained as a function of the two possible total quadrupolar parameters $G^{\mu}=\left(B^{\mu}\right)^{2} / c_{0}^{\mu}+K^{\mu}$ ( $\mu=\gamma, \varepsilon$; see Fig. 1). In addition to purely quadrupolar phases, two kinds of magnetic phases may exist: the phases $b_{Q}$ and $b_{P}$ are issued from the magnetic $\Gamma_{5}^{(1)}$ triplet ground state; in the phase $c_{Q}$, a magnetic moment is induced on the non-magnetic level, which is isolated as the ground state in the quadrupolar phase $a_{\mathrm{Q}}$.

Another interesting feature of the quadrupolar interactions is the modification of the nature of the magnetic transitions. By analyzing the free energy (Eq. (5)), the order of the transition is directly related to the sign of the following combination of the CEF susceptibilities at the critical temperature [13]:

$$
\chi_{\mathrm{M}, \mathrm{o}}^{(3)}=\chi_{\mu}^{(3)}+2 G^{\mu}\left(\chi_{\mu}^{(2)}\right)^{2} /\left(1-G^{\mu} \chi_{\mu}\right) .
$$

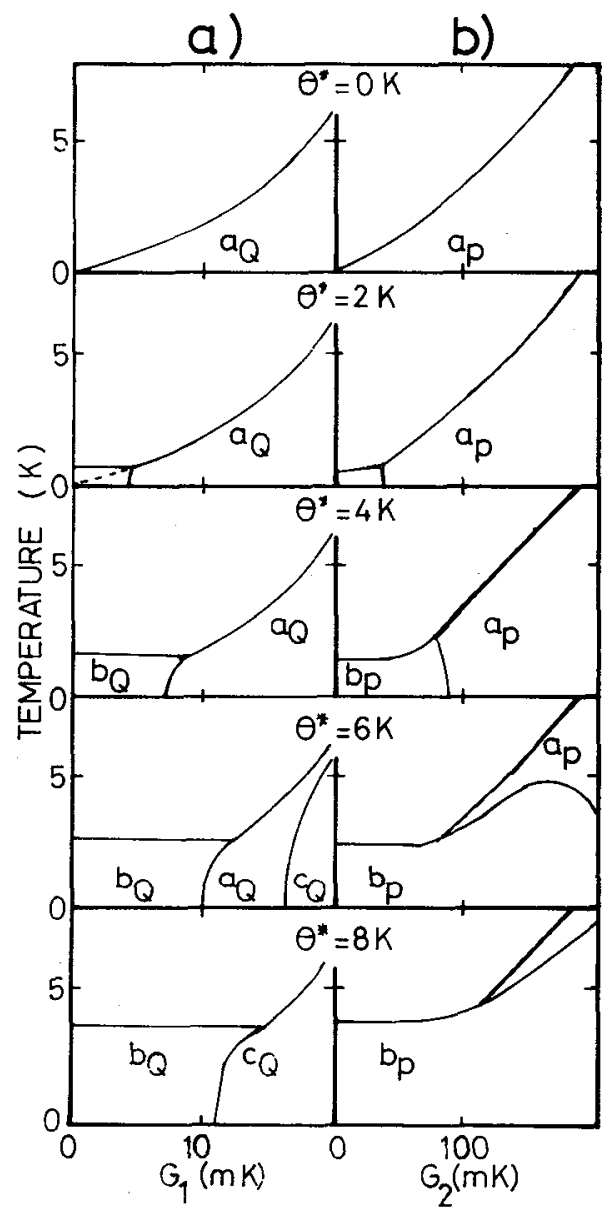

Fig. 1. - Magnetic and quadrupolar phase diagrams for various bilinear coefficients $\theta^{*} \propto n$ as a function of $G^{\gamma}=$ $G_{1}$ and $G^{\varepsilon}=G_{2}\left(\mathrm{Tm}^{3+}\right.$ case, $\left.J=6\right) \cdot a_{\mathrm{Q}}, a_{\mathrm{P}}$ : pure quadrupolar phases associated with tetragonal and trigonal symmetry, respectively; $b_{Q}, b_{P}$ : magnetic phases; $c_{Q}$ : induced magnetic phase (from Ref. [12]).

This expression includes a purely CEF term, $\chi_{\mu}^{(3)}$, and an additional quadrupolar contribution depending on the $G^{\mu \prime}$ s. A positive $G^{\mu}$ value may then drive the transition to be first-order if its contribution to $\chi_{\mathrm{M}, 0}^{(3)}$ is larger than the modulus of $\chi_{\mu}^{(3)}$, this latter term being usually negative.

This is well-illustrated in the pnictides series $\mathrm{TbX}$ [14], where the quadrupolar coupling, which increases along the sequence $\mathrm{X}=\mathrm{Bi}, \mathrm{Sb}, \mathrm{As}$ and $\mathrm{P}$, drives the antiferromagnetic ordering to be first-order for the two latter compounds $\left(\chi_{\mathrm{M}, 0}^{(3)}>0\right)$, while it is second-order for the other ones ( $\chi_{\mathrm{M}, 0}^{(3)}<0$, see Fig. 2). In particular, $\mathrm{TbSb}$ appears close to the tricriticallity $\left(\chi_{\mathrm{M}, 0}^{(3)}=0\right)$. The situation is opposite in $\mathrm{PrMg}_{2}$ compound, where the CEF term $\chi_{\gamma}^{(3)}$ is positive and where a negative 


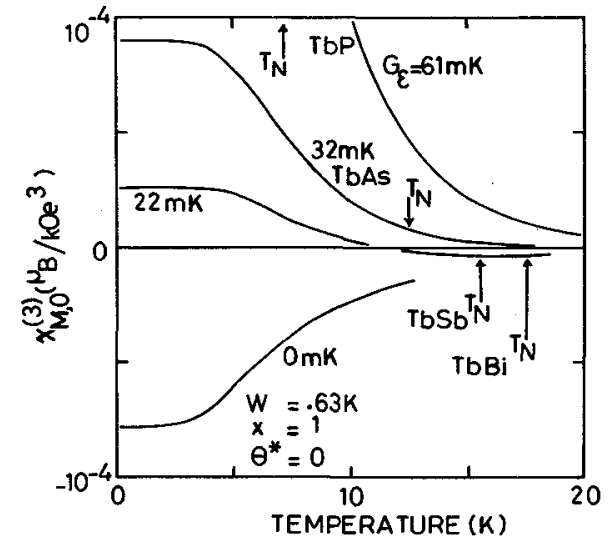

Fig. 2. - Temperature variation of $\chi_{\mathrm{M}, 0}^{(3)}$ in $\mathrm{TbX}$ pnictides in the presence of the trigonal quadrupolar parameter $G^{\varepsilon}$; the transitions at $T_{\mathrm{N}}$ are first-order in $\mathrm{TbP}, \mathrm{TbAs}$ and secondorder in TbSb, TbBi (from Ref. [1]).

$G^{\gamma}$ value leads to a negative total susceptibility $\chi_{\mathrm{M}, 0}^{(3)}$, explaining the second-order character of the transition, as observed [15].

This MFA analysis of the magnetic transitions has met a great success in many compounds as soon as quadrupolar interactions were properly taken into account.

Several other experimental manifestations of the quadrupolar interactions are directly related to the various $\chi_{\mu}^{(n)}$ 's. Indeed, by minimizing $F^{\mu}$ with regard to the variables $M, Q_{\mu}, \ldots$, one obtains relations which couple these latter with the external stresses. First, the quadrupolar field-susceptibility $\chi_{\mu}^{(2)}$ connects $Q_{\mu}$, then the strain $\varepsilon_{\mu}$, to the magnetic field:

$$
Q_{\mu}=\frac{c_{0}^{\mu}}{B^{\mu}} \quad \varepsilon_{\mu}=\frac{\chi_{\mu}^{(2)} H^{2}}{\left(1-n \chi_{0}\right)^{2}\left(1-G^{\mu} \chi_{\mu}\right)} .
$$

The associated experimental technique is called the parastriction, i.e. the measurement of the relative change of length $\lambda$ in an external magnetic field, in the paramagnetic phase [16]. A good example is provided by $\mathrm{TmZn}$ where strong anisotropic quadrupolar effects are obvious, with, in particular, large deviations in tetragonal symmetry (see Fig. 3). A spectacular situation may occur when $\chi_{\mu}^{(2)}$ changes its sign at low temperature, due to the quadrupolar character of the CEF ground state (Fig. 4): this "reverse magnetostriction" was observed for example in $\mathrm{Ce}_{x} \mathrm{La}_{1-x} \mathrm{X}$, with $\mathrm{X}=\mathrm{Sb}$ [18], Bi [19].

The strain susceptibilities $\chi_{\mu}$ represent the response of the quadrupolar moments to the corresponding stress, leading to a softening of the elastic constants through the relation:

$$
\frac{c^{\mu}}{c_{0}^{\mu}}=\frac{1-G^{\mu} \chi_{\mu}}{1-K^{\mu} \chi_{\mu}}
$$

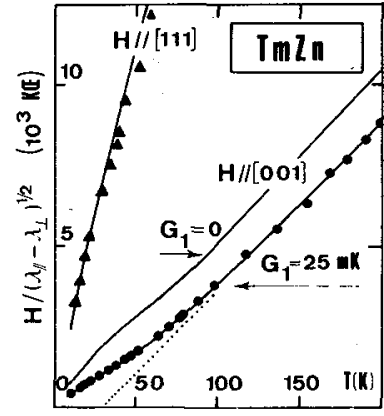

Fig. 3. - Parastriction in TmZn in tetragonal $\gamma$ and trigonal $\varepsilon$ symmetries. Curves are calculated with $B^{\gamma}=-31 \mathrm{~K}$, $G^{\gamma}=G_{1}=25 \mathrm{mK}, B^{\varepsilon}=10 \mathrm{~K}, G^{\varepsilon}=-20 \mathrm{mK}$ (from Ref. [17]).

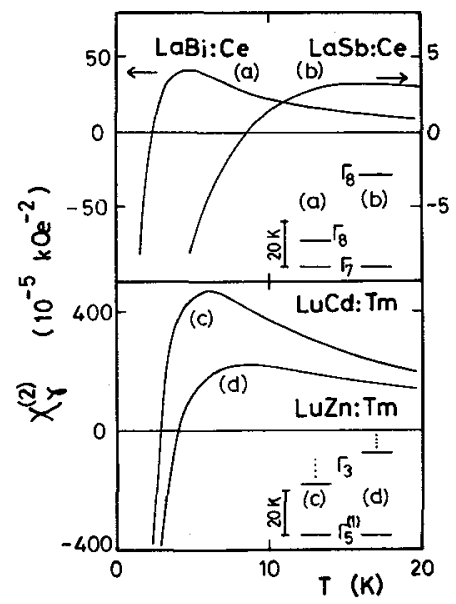

Fig. 4. - Change of sign of $\chi_{\gamma}^{(2)}$ in several systems, leading to the "reverse magnetostriction" (from Ref. [1]).

Such softenings, extensively studied in many compounds since the early 70's (see Fig. 5), constitute the most direct determination of the magnetoelastic and two-ion quadrupolar couplings because it is not perturbed by any magnetic effect which may arise in the presence of a magnetic field $[21,22]$.

A third experimental technique related to the susceptibility formalism involves the analysis of the initial curvature $\chi_{\mathrm{M}}^{(3)}$ of the magnetization curves, i.e. the third-order magnetic susceptibility:

$$
\chi_{M}^{(3)}=\frac{\chi_{M, 0}^{(3)}}{\left(1-n \chi_{0}\right)^{4}} .
$$

The anisotropic behaviour of $\chi_{M}^{(3)}$ is demonstrated in figure 6 about $\mathrm{PrPb}_{3}$.

The quadrupolar interactions may also determine the actual magnetic structure through their contribution to the free energy. In particular, the presence of 


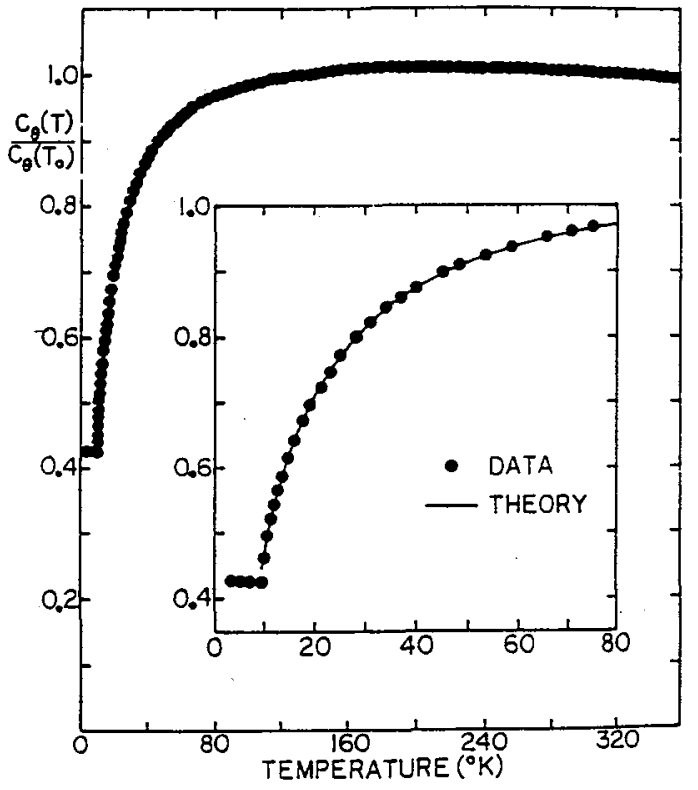

Fig. 5. - Temperature variation of $c_{\theta}=c^{\gamma}$ in DySb; line is calculated for $G^{\gamma}=1 \mathrm{mK}, K^{\gamma}=-0.4 \mathrm{mK}$ (from Ref. [20]).

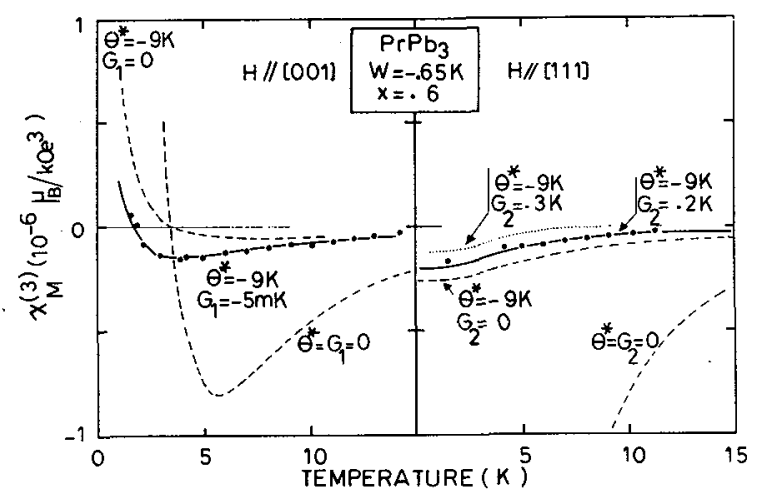

Fig. 6. - Temperature variation of $\chi_{\mathrm{M}}^{(3)}$ in $\mathrm{PrPb}_{3}$, for various quadrupolar parameters $G^{\gamma}=G_{1}, G^{\varepsilon}=G_{2}\left(\theta^{*} \propto n\right.$; from Ref. [23]).

antiferroquadrupolar (negative) interactions may stabilize multiaxial spin structures rather than collinear arrangements. This has been proved for example by neutron diffraction in magnetic field in $\mathrm{TmGa}_{3}$ (see Fig. 7). Consequently, as the quadrupolar symmetry is cubic, no spontaneous tetragonal distortion occurs in spite of a quite sizeable magnetoelastic coefficient $B^{\gamma}$.

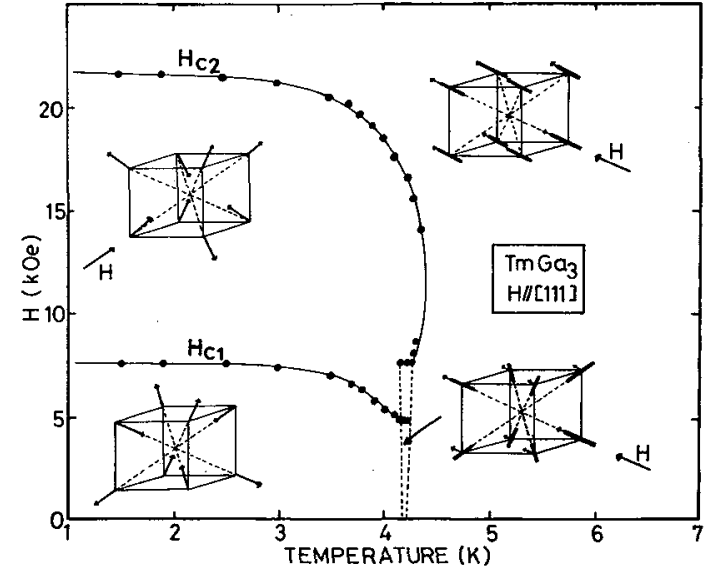

Fig. 7. - Magnetic phase diagram for a magnetic field along the [111] axis in $\mathrm{TmGa}_{3}$ (from Ref. [11]).

\section{Discussion}

The relevance of the Hamiltonian $\mathcal{H}$ has been proved in several rare earth intermetallic series by consistent determinations of the various parameters involved, from independent experimental ways [1]. The one-ion magnetoelastic coefficients deduced from the literature for cubic compounds are collected in figure 8. Their
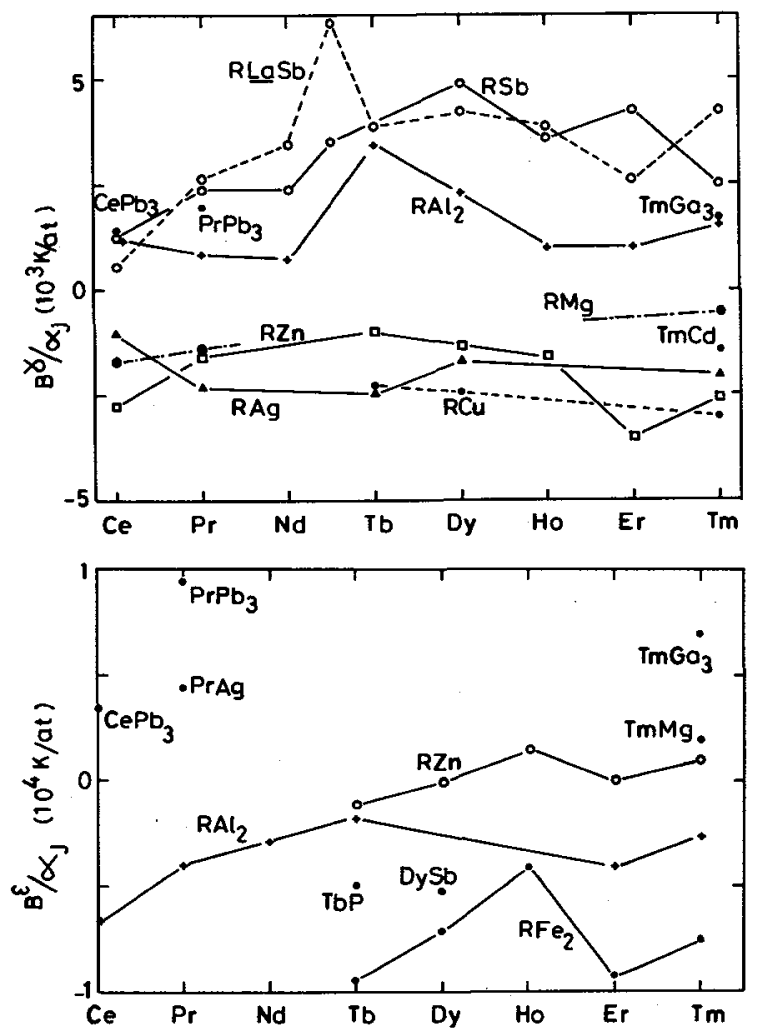

Fig. 8. - Magnetoelastic coefficients $B^{\gamma} / \alpha_{\mathbf{J}}$ (a) and $B^{\varepsilon} / \alpha_{J}(b)$ in various cubic rare-earth intermetallic series (from Ref. [1]). 
order of magnitude across a given series does not exhibit drastic variations, when they are normalized by the Stevens factor $\alpha_{J}$, particularly for the CsCl-type compounds. For a given series, the two coefficients $B^{\gamma}$ and $B^{\varepsilon}$ are of opposite sign, with only few exceptions. This suggests a dominant role for the point charge contributions to determine the magnetoelastic coupling. However, the expected point charge ratio $B^{\varepsilon} / B^{\gamma}=$ $-4 / \sqrt{3}$ is far to be satisfied. This failure, together with the detailed variation of the $B^{\mu \prime}$ s across a given series, clearly indicates the important contribution of the conduction electrons [24].

There are less systematic studies of the quadrupolar pair interactions than of the magnetoelastic coupling. In CsCl-type compounds, the tetragonal-type parameters $K^{\gamma}$ often dominate the magnetoelastic coupling and are always positive while the $K^{\varepsilon}$ is negative. On the contrary, in $\mathrm{TbX}$ pnictides, the trigonal-type coupling $K^{\varepsilon}$ is positive and dominates the one-ion magnetoelasticity. Oppositely to the Jahn-Teller compounds, where the negative two-ion quadrupolar parameters arise from a coupling via the phonons, the strong positive values observed in rare-earth intermetallics can only be attributed to a coupling mediated by the conduction electrons $[25,26]$.

From the numerous and various effects of the magnetoelastic coupling and of the two-ion quadrupolar interactions on the magnetic and elastic properties, it is clear that the existence of such couplings is quite general in rare earth intermetallic compounds, and they often need to be included to quantitatively understand the properties of these systems.

[1] Morin, P. and Schmitt, D., to appear in Ferromagnetic Materials, Eds. E. P. Wohlfarth and K. H. J. Buschow (North-Holland, Amsterdam) 1989.

[2] Hutchings, M. T., Solid. State Phys. 16 (1964) 227.

[3] Giraud, M., Morin, P. and Schmitt, D., J. Magn. Magn. Mater. 52 (1985) 41.

[4] Lüthi, B., Mullen, M. E., Andres, A., Bucher, E. and Maita, J. P., Phys. Rev. B 8 (1973) 2639.

[5] Morin, P., J. Magn. Magn. Mater. 71 (1988) 151.
[6] Ott, H. R., Andres, K., Wang, P. S., Wong, Y. H. and Lüthi, B., Crystal field effects in metals and alloys, Eds. A. Furrer (Plenum, New York) 1977, p. 84 .

[7] Bucher, E., Andres, K., Gossard, A. C. and Maita, J. P., Conf. on Low Temp. Phys., LT13, 2 (1972) 322.

[8] Peysson, Y., Ayache, C., Rossat-Mignod, J., Kunii, S. and Kasuya, T., J. Phys. France 47 (1986) 113.

[9] Czopnik, A., Iliew, N., Stalinski, B., Madge, H., Bazan, C. and Pott, R., Physica 130B (1985) 262.

[10] Effantin, J. M., Rossat-Mignod, J., Burlet, P., Bartholin, H., Kunii, S. and Kasuya, T., J. Magn. Magn. Mater. 47-48 (1985) 145.

[11] Morin, P., Giraud, M., Burlet, P. and Czopnik, A., J. Magn. Magn. Mater. 68 (1987) 107.

[12] Morin, P., Rouchy, J., Giraud, M. and Czopnik, A., J. Magn. Magn. Mater. 67 (1987) 95.

[13] Morin, P. and Schmitt, D., Phys. Rev. B 27 (1983) 4412.

[14] Kötzler, J., Z. Phys. B 55 (1984) 119.

[15] Loidl, A., Knorr, K., Mullner, M. and Buschow, K. H. J., J. Appl. Phys. 52 (1981) 1433.

[16] Morin, P., Schmitt, D. and de Lacheisserie, E., Phys. Rev. B 21 (1980) 1742.

[17] Morin, P., Schmitt, D. and de Lacheisserie, E., $J$. Magn. Magn. Mater. 15-18 (1980) 601.

[18] Nieuwenhuys, G. J., Davidov, D. and Häfner, H. U., Phys. Rev. Lett. 49 (1982) 1202.

[19] Häfner, H. U., Davidov, D. and Nieuwenhuys, G. J., J. Magn. Magn. Mater. 38 (1983) 45.

[20] Moran, T. J., Thomas, R. L., Levy, P. M. and Chen, H. H., Phys. Rev. B 7 (1973) 3238.

[21] Melcher, R. L., Physical Acoustics XII, Eds. W. P. Mason and R. N. Thurston (Academic Press, New York) 1976, p. 1.

[22] Kataoka, M. and Kanamori, J., J. Phys. Soc. Jpn 32 (1972) 113.

[23] Morin, P., Schmitt, D. and de Lacheisserie, E., J. Magn. Magn. Mater. 30 (1982) 257.

[24] Morin, P. and Schmitt, D., Phys. Rev.. B 23 (1981) 2278.

[25] Levy, P. M., Morin, P. and Schmitt, D., Phys. Rev. Lett. 42 (1979) 1417.

[26] Schmitt, D. and Levy, P. M., J. Magn. Magn. Mater. 49 (1985) 15. 